\title{
Cosmic rays and neutrino interactions beyond the standard model
}

\author{
Günter Sigl ${ }^{\mathrm{a}}$ \\ a Département d'Astrophysique Relativiste et de Cosmologie, CNRS \\ Observatoire de Paris, 92195 Meudon Cedex, France
}

Some solutions of the hierarchy problem of particle physics can lead to significantly increased neutrino cross sections beyond the electroweak scale. We discuss some consequences for and constraints resulting from cosmic ray physics.

\section{Introduction}

It has been suggested that the neutrino-nucleon cross section could be enhanced by new physics beyond the electroweak scale in the center of mass frame, or above about a $\mathrm{PeV}$ in the nucleon rest frame. A specific implementation of this possibility is given in theories with $n$ additional dimensions and a quantum gravity scale $M \sim \mathrm{TeV}$ that has recently received much attention in the literature [1] because it provides an alternative solution (i.e., without supersymmetry) to the hierarchy problem in grand unifications of gauge interactions. In such scenarios, the exchange of bulk gravitons (Kaluza-Klein modes) can lead to an extra contribution to any two-particle cross section given by [2]

$\sigma_{g} \simeq \frac{4 \pi s}{M^{4}} \simeq 10^{-27}\left(\frac{\mathrm{TeV}}{M}\right)^{4}\left(\frac{E}{10^{20} \mathrm{eV}}\right) \mathrm{cm}^{2}$,

where the last expression applies to a neutrino of energy $E$ hitting a nucleon at rest. Note that a neutrino would typically start to interact in the atmosphere and therefore become a primary candidate for the highest energy cosmic rays for $\sigma_{\nu N} \gtrsim 10^{-27} \mathrm{~cm}^{2}$, i.e. for $E \gtrsim 10^{20} \mathrm{eV}$, assuming $M \simeq 1 \mathrm{TeV}$.

The total charged-current neutrino-nucleon cross section is given by the sum of Eq. (11) and the cross section within the Standard Model, which can be estimated by [3]

$\sigma_{\nu N}^{S M}(E) \simeq 2.36 \times 10^{-32}\left(\frac{E}{10^{19} \mathrm{eV}}\right)^{0.363} \mathrm{~cm}^{2}$

in the energy range $10^{16} \mathrm{eV} \lesssim E \lesssim 10^{21} \mathrm{eV}$.
The total cross section is dominated by a contribution of the form Eq. (12) at energies $E \gtrsim E_{\mathrm{th}}$, where, for $M \gtrsim 1 \mathrm{TeV}$, the threshold energy can be approximated by

$E_{\text {th }} \simeq 2 \times 10^{13}\left(\frac{M}{\mathrm{TeV}}\right)^{6.28} \mathrm{eV}$.

This would be reflected by a linear energy dependence of the typical column depth of induced shower development if the optical depth in the detection medium is of order unity, or by a flattening of the differential detection rate by one power of the energy if the optical depth is smaller than unity. Comparison with observations would either reveal signatures for these scenarios or constrain them in a way complementary to and independent of many studies on signatures in human made accelerators 酒 or other laboratory experiments that have recently appeared in the literature.

\section{A Bound from the "Cosmogenic" Neu- trino Flux}

Fig. 1 shows neutrino fluxes for the atmospheric background at different zenith angles [5] (hatched region marked "atmospheric"), for proton blazars that are photon optically thick to nucleons [6] and whose flux was normalized to recent estimates of the blazar contribution to the diffuse $\gamma$-ray background [7] ("proton blazar"), for neutrinos created as secondaries from the decay of charged pions produced by ultra-high energy (UHE) nucleons interacting with the cosmic microwave background [8] ("cosmogenic"), and for 


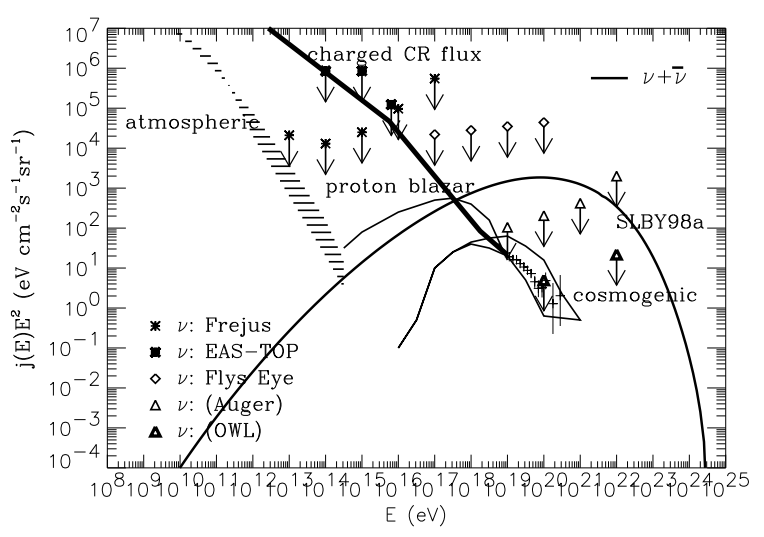

Figure 1. Predictions for the differential fluxes summed over all neutrino flavors (solid lines) from the sources discussed in the text. 1 sigma error bars are the combined data from the Haverah Park [10], the Fly's Eye [1], and the AGASA [12] experiments above $10^{19} \mathrm{eV}$. Also shown are piecewise power law fits to the observed charged CR flux (thick solid line). Points with arrows represent approximate upper limits on the diffuse neutrino flux from the Frejus [13], the EASTOP [15], and the Fly's Eye [16] experiments, as indicated. The projected sensitivity for the Pierre Auger project is using the acceptance estimated in Ref. [17], and the one for the OWL concept study is based on Ref. [20], both assuming observations over a few years period.

a model where UHE cosmic rays are produced by decay of particles close to the Grand Unification Scale ("SLBY98", see Ref. [9] for details).

Apart from the atmospheric neutrino flux only the cosmogenic neutrinos are guaranteed to exist due to the known existence of UHE cosmic rays, at least if these contain nucleons and are not exclusively of galactic origin.

The non-observation of deeply penetrating air showers by the experiments indicated in Fig. 1 in the presence of this cosmofenic flux can now be translated into an upper limit on the total neutrino-nucleon cross section $\sigma_{\nu N} \equiv \sigma_{\nu N}^{S M}+\sigma_{g}$ by scaling the diffuse neutrino flux limits from the Standard Model cross section Eq. (2). Using the conservative, lower estimate of the cosmogenic flux in Fig. 1 1 yields

$\sigma_{\nu N}\left(E=10^{19} \mathrm{eV}\right) \lesssim 2.4 \times 10^{-29} \mathrm{~cm}^{2}$,

as long as $\sigma_{\nu N}\left(E=10^{19} \mathrm{eV}\right) \lesssim 10^{-27} \mathrm{~cm}^{2}$, such that neutrinos would give rise to deeply penetrating air showers. Using Eq. (11) results in

$M \gtrsim 1.4 \mathrm{TeV}$.

It is interesting to note that these limits do not depend on the number $n$ of extra dimensions, in contrast to some other astrophysical limits such as from graviton emission from a hot supernova core into the extra dimensions which depend more explicitly on phase space integrations (see Sect. 3 below).

As can be seen from Fig. 1, with an experiment such as OWL, the upper limit on the cross section Eq. (4) could improve by about 4 orders of magnitude, and the lower limit on $M$ consequently by about a factor 10 .

\section{Comparison with Other Astrophysical and Laboratory Bounds}

There are also astrophysical constraints on $M$ which result from limiting the emission of bulk gravitons into the extra dimensions. The strongest constraints in this regard come from nucleon-nucleon bremsstrahlung in type II supernovae 21. These contraints read $M \gtrsim 50 \mathrm{TeV}$, $M \gtrsim 4 \mathrm{TeV}$, and $M \gtrsim 1 \mathrm{TeV}$, for $n=2,3,4$, respectively, and, therefore, $n \geq 4$ is required if neutrino primaries are to serve as a primary candidate for the UHE cosmic ray events observed above $10^{20} \mathrm{eV}$ (note that $n=7$ for the superstring and $n=22$ for the heterotic string). This assumes that all extra dimensions have the same size given by

$$
\begin{aligned}
r & \simeq M^{-1}\left(\frac{M_{\mathrm{Pl}}}{M}\right)^{2 / n} \\
& \simeq 2 \times 10^{-17}\left(\frac{\mathrm{TeV}}{M}\right)\left(\frac{M_{\mathrm{Pl}}}{M}\right)^{2 / n} \mathrm{~cm},
\end{aligned}
$$

where $M_{\mathrm{Pl}}$ denotes the Planck mass. The above lower bounds on $M$ thus translate into the cor- 
responding upper bounds $r \lesssim 3 \times 10^{-4} \mathrm{~mm}, r \lesssim$ $4 \times 10^{-7} \mathrm{~mm}$, and $r \lesssim 2 \times 10^{-8} \mathrm{~mm}$, respectively.

UHE cosmic rays and neutrinos together with other astrophysical and cosmological constraints thus provide an interesting testing ground for theories involving extra dimensions which represent one possible kind of physics beyond the Standard Model. In this context, we mention that in theories with large compact extra dimensions mentioned above, Newton's law of gravity is expected to be modified at distances smaller than the length scale given by Eq. (6). Indeed, there are laboratory experiments measuring gravitational interaction at small distances (for a recent review of such experiments see Ref. [22]), which also probe these theories. Thus, future UHE cosmic ray experiments and gravitational experiments in the laboratory together have the potential of providing rather strong tests of these theories. These tests would be complementary to constraints from collider experiments 陆.

\section{REFERENCES}

1. N. Arkani-Hamed, S. Dimopoulos, and G. Dvali, Phys. Lett. B 429 (1998) 263; I. Antoniadis, N. Arkani-Hamed, S. Dimopoulos, and G. Dvali, Phys. Lett. B 436 (1998) 257; N. Arkani-Hamed, S. Dimopoulos, and G. Dvali, Phys. Rev. D 59 (1999) 086004.

2. S. Nussinov and R. Shrock, Phys. Rev. D 59 (1999) 105002.

3. R. Gandhi, C. Quigg, M. H. Reno, and I. Sarcevic, Astropart. Phys. 5 (1996) 81; Phys. Rev. D 58 (1998) 093009.

4. see, e.g., T. G. Rizzo and J. D. Wells, Phys. Rev. D 61 (2000) 016007, and references therein.

5. P. Lipari, Astropart. Phys. 1 (1993) 195.

6. R. Protheroe, in Accretion Phenomena and Related Outflows, Vol. 163 of IAU Colloquium, eds. D. Wickramasinghe, G. Bicknell, and L. Ferrario (Astron. Soc. of the Pacific, 1997), p. 585.

7. R. Mukherjee and J. Chiang, Astropart. Phys. 11 (1999) 213.

8. R. J. Protheroe and P. A. Johnson, Astropart. Phys. 4 (1996) 253, and erratum ibid.
5 (1996) 215.

9. G. Sigl, S. Lee, P. Bhattacharjee, and S. Yoshida, Phys. Rev. D 59 (1999) 043504.

10. See, e.g., M. A. Lawrence, R. J. O. Reid, and A. A. Watson, J. Phys. G Nucl. Part. Phys. 17 (1991) 733, and references therein; see also http://ast.leeds.ac.uk/haverah/hav-home .html.

11. D. J. Bird et al., Phys. Rev. Lett. 71 (1993) 3401; Astrophys. J. 424 (1994) 491; ibid. 441 (1995) 144.

12. N. Hayashida et al., Phys. Rev. Lett. 73 (1994) 3491; S. Yoshida et al., Astropart. Phys. 3 (1995) 105; M. Takeda et al., Phys. Rev. Lett. 81 (1998) 1163; see also http://icrsun.icrr.u-tokyo.ac.jp/as/project /agasa.html.

13. W. Rhode et al., Astropart. Phys. 4 (1996) 217.

14. Proc. 24th International Cosmic Ray Conference (Istituto Nazionale Fisica Nucleare, Rome, Italy, 1995)

15. M. Aglietta et al. (EAS-TOP collaboration), in [14], Vol. 1, 638.

16. R. M. Baltrusaitis et al., Astrophys. J. 281 (1984) L9; Phys. Rev. D 31 (1985) 2192.

17. J. J. Blanco-Pillado, R. A. Vázquez, and E. Zas, Phys. Rev. Lett. 78 (1997) 3614; K. S. Capelle, J. W. Cronin, G. Parente, and E. Zas, Astropart. Phys. 8 (1998) 321.

18. Proc. 25th International Cosmic Ray Conference, eds.: M. S. Potgieter et al. (Durban, 1997).

19. Proc. of International Symposium on Extremely High Energy Cosmic Rays: Astrophysics and Future Observatories, ed. M. Nagano (Institute for Cosmic Ray Research, Tokyo, 1996).

20. J. F. Ormes et al., in 18], Vol. 5, 273; Y. Takahashi et al., in 19], p. 310; see also http://lheawww.gsfc.nasa.gov/docs/gamcosray /hecr/OWL//.

21. S. Cullen and M. Perelstein, Phys. Rev. Lett. 83 (1999) 268; V. Barger, T. Han, C. Kao, and R.-J. Zhang, Phys. Lett. B 461 (1999) 34.

22. J. C. Long, H. W. Chan, and J. C. Price, Nucl. Phys. B 539 (1999) 23. 